\title{
A CROSS-SECTIONAL ANALYSIS OF APOE GENE POLYMORPHISM AND THE RISK OF COGNITIVE IMPAIRMENTS IN THE ALZHEIMER'S DISEASE NEUROIMAGING INITIATIVE STUDY
}

\author{
G. Wang ${ }^{1}$, D.E. Vance ${ }^{2}$, W. Li $i^{3}$, for the Alzheimer's Disease Neuroimaging Initiative*
}

\begin{abstract}
Background: It is inconclusive on how apolipoprotein epsilon (APOE) gene polymorphism is associated with the risk of having mild cognitive impairment (MCI) or Alzheimer's disease (AD). Objectives: To investigate how APOE genotype is associated with the risk of MCI or AD using the data collected from the Alzheimer's Disease Neuroimaging Initiative (ADNI) participants. Methods: A cross-sectional design was used to analyze the baseline data collected from the 1,720 ADNI participants. APOE gene polymorphism was analyzed on how they are related to the risk of cognitive impairments of either MCI or AD using a percent yield (PY) method. Then cognitive functions were compared among six different APOE genotypes using a two-way ANCOVA by controlling possible confounding factors. Results: The prevalence of six APOE genotypes in 1,720 participants is as following: e2/e2 (0.3\%), e2/ e3 (7.4\%), e3/e3 (45.4\%), e2/e4 (2\%), e3/e4 (35\%) and e4/e4 (9.9\%). The e2/e2 and e4/e4 genotypes were associated with the lowest and the highest risk respectively for cognitive impairments of either MCI or AD. Further, a worse cognitive diagnosis was associated with an increasing number of APOE e4 allele in a dose dependent manner. Participants with genotype e3/e3 had a better memory measure than those with the genotype of e3/e4. Conclusions: APOE gene polymorphism is associated with different level of risks for cognitive impairments. The heterozygous genotype e3/e4 is associated with a worse memory function compared to the genotype of e3/e3. Further investigations are needed to intervene the cognitive deteriorations in those with at risk APOE genotypes.
\end{abstract}

Key words: Alzheimer's disease (AD), apolipoprotein epsilon (APOE), mild cognitive impairment (MCI), aging, lifestyle.

In 1993, the apolipoprotein epsilon (APOE) gene polymorphism was reported to be associated with the risk of late-onset Alzheimer's disease (AD) (1). APOE, the major susceptibility gene for late-onset, sporadic $\mathrm{AD}$, is located on chromosome $19 \mathrm{q} 13.2$ (2). There are three common APOE alleles: e2, e3, and e4, which are defined by two single nucleotide polymorphisms in APOE (rs429358/e4, rs7412/e2) (3). As a result, there are six different APOE genotypes: e2/e2, e2/e3, e2/e4, e3/ e3, e3/e4, and e4/e4. Three of them are homozygous (e2/e2, e3/e3, and e4/e4) and the remaining three are heterozygous (e2/e3, e2/e4, and e3/e4) genotypes. In 1994, allele e2 was demonstrated to have protective effects against $\mathrm{AD}(4)$. By contrast, allele e4 could increase the risk of sporadic AD in a dose dependent manner (4). In 2003, one study confirmed the association between

1. Tongji Hospital, Tongji Medical College, Huazhong University of Science and Technology, Wuhan, China; 2. Office of Research and Scholarship, School of Nursing, University of Alabama at Birmingham, Birmingham, Alabama; 3. Physician Assistant Studies, School of Health Professions, University of Alabama at Birmingham, Birmingham, Alabama

Corresponding Author: Dr. Wei Li, SHPB 485, 1720 2nd Avenue South, Birmingham, AL 35294, USA, Phone: 205-996-2656, Fax: 205-975-7302, Email: wli@uab.edu

Received March 4, 2021

Accepted for publication March 12, 2021 allele e4 and the risk of AD (5). In 2018, AD risk was shown to be increased with APOE genotype varying from e2/e3 to e2/e4 to e3/e3 to e3/e4 to e4/e4 in a population-based cohort study (6). However, it is still not clear how APOE genotype is associated with the risk of mild cognitive impairment $(\mathrm{MCI})$ and memory function. Using the data collected from the Alzheimer's Disease Neuroimaging Initiative (ADNI), the purpose of this secondary data analysis study was to categorize how APOE genotype is associated with: 1) the risk of MCI or $\mathrm{AD}$; and 2) cognitive performance. Interestingly, the APOE gene polymorphism was shown to correlate with the outcome in 58 individuals with mild-to-moderate $\mathrm{AD}$ after a 10 -weeks-long multidimensional stimulation therapy (7). The ADNI participants represent an elderly group who are or were living a relatively healthy lifestyle. The potential findings of our study would be meaningful to direct the care for those with cognitive impairments based on the information of APOE gene polymorphism and cognitive status (MCI or AD). For example, besides living a healthy lifestyle, the clinical management of individuals with cognitive impairments can be optimized by considering the current cognitive diagnosis and the 
risk of cognitive deterioration associated with a certain APOE genotype.

\section{Methods}

\section{$A D N I$}

Data were downloaded from the ADNI database (adni. loni.usc.edu) on October 6, 2019. As an ongoing project, ADNI was launched in 2003 and have been sponsored by the following agencies: National Institute on Aging (NIA), National Institute of Biomedical Imaging and Bioengineering (NIBIB), Food and Drug Administration (FDA), private pharmaceutical companies, and nonprofit organizations. The primary goal of the ADNI has been to test whether serial magnetic resonance imaging (MRI), positron emission tomography (PET), biomarkers, and clinical and neuropsychological assessment can be combined to measure the progression of $\mathrm{MCI}$ and early $\mathrm{AD}$ (8). In the first three phases $(1, \mathrm{GO}$, and 2$)$, the ADNI recruited over 1,700 adult participants from over 50 sites across the United States and Canada. The participants were people (55 to 90 years old), and they consisted of people with different cognitive diagnosis at the baseline visit. Further information about this parent study can be found at http:/ / www.adni-info.org/ and in previous reports (8-13).

\section{APOE Genotyping}

APOE genotyping was done using DNA from blood samples collected from ADNI participants. For ADNI1 participants, APOE genotyping was done through polymerase chain reaction (PCR) amplification, Hhal restriction enzyme digestion, and subsequent standard gel resolution processes $(14,15)$. For ADNI-GO and ADNI-2 participants, genotyping was carried out by Prevention Genetics and LGC Genomics. Prevention Genetics employed array processing using allele-specific PCR with universal molecular beacons $(16,17)$. At LGC, assays were performed using competitive allele-specific PCR, enabling bi-allelic scoring of single nucleotide polymorphisms. Genotypes were called and returned to the ADNI Genetics Core after manual quality control.

\section{Baseline Cognitive Diagnosis}

For ADNI phase 1, participants were recruited with three cognitive diagnoses at baseline: healthy control (HC), MCI, and AD. The recruitment criteria for $\mathrm{HC}$ participants included MMSE scores between 24-30 (inclusive), a clinical dementia rating (CDR) of 0 , nondepressed, no diagnosis of either MCI or dementia. The recruitment criteria for participants with MCI included Mini Mental Status Examination (MMSE) scores between 24-30 (inclusive), a memory complaint, have objective memory loss measured by education adjusted scores on
Wechsler Memory Scale Logical Memory II, a CDR of 0.5 , absence of significant levels of impairment in other cognitive domains, essentially preserved activities of daily living, and an absence of dementia. The recruitment criteria for participants with AD included MMSE scores between 20-26 (inclusive), CDR of 0.5 or 1.0, and meeting NINCDS / ADRDA criteria for probable AD.

For phases GO and 2, the diagnosis of MCI was separated into early $\mathrm{MCI}$ (EMCI) and late $\mathrm{MCI}$ (LMCI). The criteria for EMCI were: MMSE scores between 24-30 (inclusive), a memory complaint (reported by subject or informant), must have objective memory loss measured by education adjusted scores on delayed recall of one paragraph from Wechsler Memory Scale Logical Memory II (between approximately 0.5 and $1.5 \mathrm{SD}$ below the mean of Cognitively Normal), a CDR of 0.5 , absence of significant levels of impairment in other cognitive domains, essentially preserved activities of daily living, and an absence of dementia.

For phase 2, significant memory concern (SMC) was added as one separate category of baseline cognitive diagnosis. Participants with SMC had self-reported memory concern, quantified by using the Cognitive Change Index and the CDR of Zero. However, they scored normally for cognitive tests, and the informant did not equate the expressed concern with progressive memory impairment.

The detailed information on baseline cognitive diagnosis and APOE genotype was provided in Table 1 for the 1,720 ADNI participants. Since cognitive diagnoses of SMC, EMCI, and LMCI were added after the ADNI phase 1, participants with cognitive diagnoses of SMC and $\mathrm{HC}$ were combined into one group: Cognitively normal $(\mathrm{CN})$ for our data analysis purpose. As such, $\mathrm{EMCI}$ and $\mathrm{LMCI}$ were also combined into the MCI group (Table 1).

\section{Cognitive Measures}

The cognitive assessment raw data from the baseline visit were processed and converted into composite scores using validated methods (18-20). The ADNI participants had a comprehensive neuopsychological assessment at the baseline. Individual tests were chosen for analyzing cognitive functions in domains of executive function, language, memory, and visuospatial function. A bi-factor model was used to calculate a composite score for each of the cognitive functions defining the mean at 0 and standard deviation of 1. A lower composite score corresponds with a worse cognitive performance in each of the cognitive domains.

\section{Data Analysis}

For calculating the relative risk of each APOE genotype associated with cognitive impairment of MCI or AD, we used a method called percent yield (PY) (21). For 
Table 1

Demographic information and baseline cognitive diagnosis were compared among groups of participants with different APOE genotypes

\begin{tabular}{lccccccc}
\hline & e2/e2 & e2/e3 & e3/e3 & e2/e4 & e3/e4 & e4/e4 & Sum \\
\hline CN & 3 & 68 & 301 & 7 & 130 & 12 & $521(30.3 \%)$ \\
MCI & 2 & 55 & 413 & 23 & 382 & 121 & $996(57.9 \%)$ \\
AD & 0 & 5 & 66 & 4 & 90 & 38 & $203(11.8 \%)$ \\
Sex (M) & $40 \%$ & $52.3 \%$ & $55.1 \%$ & $44.1 \%$ & $55.3 \%$ & $59.1 \%$ & \\
Race (White) & $40 \%$ & $92.2 \%$ & $92.6 \%$ & $85.3 \%$ & $93.5 \%$ & $92.4 \%$ & \\
Age & $76.40 \pm 3.19$ & $73.75 \pm 0.63$ & $74.10 \pm 0.26$ & $74.09 \pm 1.22$ & $73.04 \pm 0.29$ & $70.53 \mathrm{v} 0.55$ & \\
Education & $17.00 \pm 1.28$ & $16.00 \pm 0.25$ & $16.08 \pm 0.10$ & $15.88 \pm 0.49$ & $15.73 \pm 0.12$ & $15.71 \pm 0.22$ & \\
\hline
\end{tabular}

AD: Alzheimer's disease; APOE = Apolipoprotein Epsilon; $\mathrm{CN}$ : Cognitively normal; $\mathrm{M}$ = male; MCI: mild cognitive impairment

Table 2

The risk of cognitive impairments shown as percent yield was associated with APOE genotype

\begin{tabular}{lcccccc}
\hline & e2/e2 & e2/e3 & e3/e3 & e2/e4 & e3/e4 & e4/e4 \\
\hline CN & 1.98 & 1.75 & 1.27 & 0.68 & 0.71 & 0.23 \\
MCI & 0.69 & 0.74 & 0.91 & 1.17 & 1.10 & 1.22 \\
AD & 0.00 & 0.33 & 0.72 & 1.00 & 1.27 & 1.88 \\
\hline
\end{tabular}

$\mathrm{AD}=$ Alzheimer's disease $\mathrm{CN}$ = cognitively normal; $\mathrm{MCI}=$ mild cognitive impairment

the ADNI, all participants were recruited into the study based on their clinical diagnosis of CN, MCI, and AD. The enrollment ratios of these three baseline diagnoses of $\mathrm{CN}$, $\mathrm{MCI}$, and AD were $30.3 \%, 57.9 \%$, and $11.8 \%$ respectively (Table 1).

For each APOE genotype, the PY was calculated with dividing the actual count of participants with each cognitive diagnosis ( $\mathrm{CN}, \mathrm{MCI}$, or $\mathrm{AD})$ by the theoretical allocation based on the enrollment ratio and the total count of participants for each APOE genotype. For example, there were 5 participants with the genotype e2/e2. Based on the enrollment ratio, there should be $30.3 \% * 5=1.51$ persons being allocated to the CN group. Actually, there were 3 participants with the genotype e2/ e2 and who were also cognitively normal. Therefore, the PY is 3/1.51=1.98 for participants with the genotype e2/ e2, who were cognitive normal (Table 2).

SPSS (version 26.0) was used to conduct all statistical analyses. A one-way analysis of variance (ANOVA) was used to compare age at baseline or education among the six APOE genotype groups (Table 3). Chisquare tests were used to examine the relationship of the APOE genotype with either sex or race (Table 3). Then a two-way analysis of covariance (ANCOVA) model was utilized to evaluate how APOE genotype interacts with baseline diagnostic group (CN, MCI, AD) to affect the cognitive functions with controlling age at baseline, gender, education, and race. Bonferroni posthoc correction was used for comparing the cognitive functions across the six APOE genotype groups.
Data were shown in the form of mean \pm standard deviation for both age and education, and $\mathrm{p}<0.05$ was considered as significant for all statistical analyses.

\section{Data Availability Statement}

Data and analytical methods are carefully documented for the performed study. Any data-sharing request can only be submitted to the ADNI for approval purpose.

\section{Results}

For the 1,720 ADNI study participants with their APOE genotypes determined, e3/e3 (45.4\%) and e3/e4 (35\%) were most commonly seen (Table 1 ). By contrast, the homozygous e2/e2 genotype was the least common $(0.3 \%)$. The second least common seen genotype was e2/ e4 $(2 \%)$. The percentages for genotypes of e2/e3 and e4/ e4 were $7.4 \%$ and $9.9 \%$, respectively (Table 1 ).

At baseline, participants from different APOE genotype groups were significantly different pertaining to either age or race but not education or sex (Table 1). For the baseline age in years, participants in the e4/ e4 group had an average age of $70.53 \pm 0.55$, which was significantly younger than that for the e2/e3 group of $73.75 \pm 0.63(\mathrm{p}=0.002)$ or $\mathrm{e} 3 / \mathrm{e} 3$ of $74.10 \pm 0.26$ $(\mathrm{p}<0.001)$. For race, most APOE genotype groups were composed of mainly Whites except the e2/e2 group, which had two Whites out of a total of five participants.

For $\mathrm{AD}$, the relative risk (RR) was in an increasing 
Table 3

Cognitive functions were compared among participant groups with different APOE genotypes

\begin{tabular}{lcccccc}
\hline & e2/e2 & e2/e3 & e3/e3 & e2/e4 & e3/e4 & e4/e4 \\
\hline Executive Function & $-1.2 \pm 0.43$ & $0.21 \pm 0.1$ & $0.19 \pm 0.04$ & $-0.05 \pm 0.17$ & $-0.08 \pm 0.04$ & $0.03 \pm 0.18$ \\
Language & $-0.38 \pm 0.39$ & $0.22 \pm 0.09$ & $0.19 \pm 0.03$ & $0.33 \pm 0.16$ & $0.16 \pm 0.04$ & $0.17 \pm 0.17$ \\
Memory & $0.42 \pm 0.3$ & $0.31 \pm 0.07$ & $0.37 \pm 0.03$ & $0.33 \pm 0.12$ & $0.23 \pm 0.03$ & $0.16 \pm 0.03$ \\
Visuospatial Function & $-0.97 \pm 0.38$ & $-0.09 \pm 0.09$ & $-0.08 \pm 0.03$ & $-0.14 \pm 0.15$ & $-0.11 \pm 0.04$ & $0.08 \pm 0.16$ \\
\hline
\end{tabular}

trajectory in the order of e2/e2, e2/e3, e3/e3, e2/e4, e3/ e4, and e4/e4. Genotype e3/e4 had a RR of 1.27 , which was lower than the same measure for the e4/e4 group of 1.88 (Table 2). Interestingly, genotype e2/e4 did not increase the $\mathrm{AD}$ risk with the RR of 1 . For MCI, the RRs for e2/e4, e3/e4, and e4/e4 were 1.17, 1.10, and 1.22, respectively.

At last, cognitive functions were compared among the six APOE genotype groups (Table 3). For the executive function, the APOE gene polymorphism had significant effects $(\mathrm{F}=2.97, \mathrm{p}=0.011)$ with the e2/e2 group had lower a low composite score than the same measure for the group of e2/e3, e3/e3 or e3/e4. Similarly, there was a significant effect of APOE gene polymorphism on the memory function $(\mathrm{F}=2.75, \mathrm{p}=0.018)$. The e3/e3 group had a mean memory composite score of $0.37 \pm 0.03$ ( $\mathrm{N}=780,95 \%$ confidence interval (CI): 0.32-0.42), which was significantly higher than the same measure for the e3/e4 group of $0.23 \pm 0.03$ (95\% CI: $0.17-0.29, \mathrm{~N}=602$, $\mathrm{p}=0.007)$ (Table 3). By contrast, neither language $(\mathrm{F}=0.75$, $\mathrm{p}=0.59)$ nor visuospatial function $(\mathrm{F}=1.38, \mathrm{p}=0.23)$ were significantly different among the six different APOE genotype groups (Table 3 ).

\section{Discussion}

Out of the three APOE alleles: e2, e3, and e4, the e3 allele is the most common one followed by e4 then e2 (2), which is consistent with our observations from the ADNI data as e3/e3 and e3/e4 are the most commonly seen genotypes (Table 1). The most common e3 allele was considered to be neutral regarding to AD risk. By contrast, the e2 allele was considered to be protective and associated with a lower risk of AD (4). In addition, each additional copy of APOE e4 was associated with a higher risk and younger age at onset for late onset$\mathrm{AD}(4)$. As expected, the e2/e2 genotype had the most protective effects against MCI or AD. However, the genotype e2/e2 was reported for having a slightly higher 10 -year absolute risk for $\mathrm{AD}$ than that for the genotype of e2/e3 (6). For either MCI or AD, both e2/e3 and e3/ e3 genotypes had protective roles as shown in Table 2 . Interestingly, the e2/e4 genotype did not increase the risk for AD but did so for MCI with a PY of 1.17 (Table 2). As expected, both e3/e4 and e4/e4 genotypes were associated with an increased risk for either MCI or AD. A dose dependent effect was observed for the e4 allele for being a risk factor for $\mathrm{AD}$ as reported previously (22). It was also reported that e4 allele was associated with an increased risk and e2 allele had a protective role for AD development (23). In another study, individuals with subjective cognitive decline were also shown to have a higher e4 allele frequency than the controls (24).

The e2/e2 had worse executive functions than the genotype groups of e2/e3, e3/e3, or e3/e4, which was unexpected and the findings might be skewed due to the small sample size of the e2/e2 group $(n=5)$. The e3/e3 group had a better performance than the e3/ e4 group on memory, which indicated allele e4 had deteriorative effects on this cognitive function (Table 3). It was reported that the protective effects of allele e2 on memory was only observed in females and the effects of allele e4 were unobserved (25).

The e4/e4 group had a significantly younger baseline age than all other groups. Plus, the e4/e4 group had the worst memory performance among all APOE genotype groups (Table 26). In a previous report, even for participants with normal cognitive functions, the e4 allele carriers had a faster declining rate of cognitive performance than the non-carriers (26).

The relation between APOE gene polymorphism and cognitive function had been studied before. For example, individuals carrying the e4 allele showed contextual cueing deficits compared to those who did not carry the e4 allele (27). In addition, an APOE genotype containing e4 allele was shown as an independent risk factor for cognitive decline from a longitudinal study of 14 years (28). In participants with MCI, APOE e4 carrier genotype was associated with a poorer frontal executive function than the e4 non-carrier genotype (29). Further, performance on the MMSE was significantly poorer for e4/e4 homozygotes than e4 heterozygotes or e4 non-carriers (30). Structurally, an e4 allele dose effect was observed for accelerating hippocampal atrophy in participants with different baseline cognitive diagnosis (31). In the current report, we observed the deteriorative effect from the e 4 allele but not the protective effect from the e2 allele for the memory function.

Our study had some limitations. The number of participants were small for some genotype groups. For example, there were only 5 participants in the 
genotype e2/e2 group. In addition, the e2/e4 group had 34 participants. The ADNI participants were mainly composed of Whites and recruited based on the baseline cognitive diagnosis for a prospective cohort study. The participants are generally well educated, have a decent socioeconomic status, and living a healthy lifestyle. Therefore, it is worthy to note that our study was not based on a randomly selected, population-based sample, which could limit the generality of our findings.

In conclusion, our findings supported the e2 allele had protective role for reducing the risk for $\mathrm{MCI}$ or $\mathrm{AD}$. At the same time, the data provided evidence on the e4 allele's deteriorative role for cognitive impairments in memory. However, our findings have the following implications for the elderly population especially for those with at risk APOE genotypes. First, it might be crucial to practice precision medicine using cognitive stimulation training to help those who already developed or are at risk for developing cognitive impairments based on their genetic information. For example, the e4 allele carriers should be monitored closely on cognitive impairment appearance and given corresponding stimulation training/treatment. Second, the APOE gene polymorphism is more closely associated with cognitive performance in some domains (for example memory) than others. Thus, it is important to consider the gene polymorphism factor for doing cognitive stimulation therapy targeting on a specific domain as other researchers begun to consider (32). At last, adopting or maintaining a healthy lifestyle is important to reduce the risk of cognitive impairments despite the genetic predisposition.

*Data used in preparation of this article were obtained from the Alzheimer's Disease Neuroimaging Initiative (ADNI) database (adni.loni.usc.edu). As such, the investigators within the ADNI contributed to the design and implementation of ADNI and/or provided data but did not participate in analysis or writing of this report. A complete listing of ADNI investigators can be found at: http: / / adni.loni. usc.edu/wp- content/uploads/how_to_apply/ ADNI_Acknowledgement_ List. pdf

Author contributions: All authors contributed to data analysis and interpretation, draft and critical revision of the manuscript for important intellectual content.

\section{Conflict of interest: All authors have nothing to disclose.}

Ethical standard: Written informed consent was obtained from all participants (or guardians of participants) participating in the study (consent for research). The IRB approval was also obtained from each participating clinical/ research site.

Acknowledgments: The NACC database is funded by NIA/NIH Grant U01 AG016976. NACC data are contributed by the NIA-funded ADCs: P30 AG019610 (PI Eric Reiman, MD), P30 AG013846 (PI Neil Kowall, MD), P50 AG008702 (PI Scott Small, MD), P50 AG025688 (PI Allan Levey, MD, PhD), P50 AG047266 (PI Todd Golde, MD, PhD), P30 AG010133 (PI Andrew Saykin, PsyD), P50 AG005146 (PI Marilyn Albert, PhD), P50 AG005134 (PI Bradley Hyman, MD, PhD), P50 AG016574 (PI Ronald Petersen, MD, PhD), P50 AG005138 (PI Mary Sano, PhD), P30 AG008051 (PI Thomas Wisniewski, MD), P30 AG013854 (PI M. Marse Mesulam, MD), P30 AG008017 (PI Jeffrey Kaye, MD), P30 AG010161 (PI David Bennett, MD), P50 AG047366 (PI Victor Henderson, MD, MS), P30 AG010129 (PI Charles DeCarli, MD), P50 AG016573 (PI Frank LaFerla, PhD), P50 AG005131 (PI James Brewer, MD, PhD), P50 AG023501 (PI Bruce Miller, MD), P30 AG035982 (PI Russell Swerdlow, MD), P30 AG028383 (PI Linda Van Eldik, PhD), P30 AG053760 (PI Henry Paulson, MD, PhD), P30 AG010124 (PI John Trojanowski, MD, PhD), P50 AG005133 (PI Oscar Lopez, MD), P50 AG005142 (PI Helena Chui, MD), P30 AG012300 (PI Roger Rosenberg, MD), P30 AG049638 (PI Suzanne Craft, PhD), P50 AG005136 (PI Thomas Grabowski, MD), P50 AG033514 (PI Sanjay Asthana MD, FRCP), P50 AG005681 (PI John Morris, MD), P50 AG047270 (PI Stephen
Strittmatter, MD, $\mathrm{PhD}$ ).

\section{References}

1. Corder EH, Saunders AM, Strittmatter WJ, et al. Gene dose of apolipoprotein E type 4 allele and the risk of Alzheimer's disease in late onset families. Science 1993; 261:921-923.

2. Giau VV, Bagyinszky E, An SS, Kim SY. Role of apolipoprotein E in neurodegenerative diseases. Neuropsychiatr Dis Treat 2015; 11:1723-1737.

3. Sidaraite A, Vilkeviciute A, Glebauskiene B, Kriauciuniene L, Zaliuniene D, Liutkeviciene R. Association of ApoE haplotype with clinical evidence of pituitary adenoma. Gene 2019; 706:154-161.

4. Corder EH, Saunders AM, Risch NJ, et al. Protective effect of apolipoprotein E type 2 allele for late onset Alzheimer disease. Nat Genet 1994; 7:180-184.

5. Engelborghs S, Dermaut B, Goeman J, et al. Prospective Belgian study of neurodegenerative and vascular dementia: APOE genotype effects. J Neurol Neurosurg Psychiatry 2003; 74:1148-1151.

6. Rasmussen KL, Tybjærg-Hansen A, Nordestgaard BG, Frikke-Schmidt R (2018) Absolute 10-year risk of dementia by age, sex and APOE genotype: a population-based cohort study. CMAJ 2018; 190:E1033-E1041.

7. Guerini FR, Farina E, Costa AS, et al. ApoE and SNAP-25 polymorphisms predict the outcome of multidementional stimulation therapy rehabilitation in Alzheimer's Disease. Neurorehabil Neural Repair 2016; 30: 883-893.

8. Weiner MW, Aisen PS, Jack CR Jr, et al. The Alzheimer's disease neuroimaging initiative: progress report and future plans. Alzheimers Dement 2010; 6:202-211.e7.

9. Jagust WJ, Bandy D, Chen K, et al. The Alzheimer's Disease Neuroimaging Initiative positron emission tomography core. Alzheimers Dement 2010; 6:221-229.

10. Petersen RC, Aisen PS, Beckett LA, et al. Alzheimer's Disease Neuroimaging Initiative (ADNI): clinical characterization. Neurology 2010; 74:201-209.

11. Saykin AJ, Shen L, Foroud TM, et al. Alzheimer's Disease Neuroimaging Initiative biomarkers as quantitative phenotypes: genetics core aims, progress, and plans. Alzheimers Dement 2010; 6:265-273.

12. Trojanowski JQ, Vandeerstichele H, Korecka M, et al. Update on the biomarker core of the Alzheimer's Disease Neuroimaging Initiative subjects. Alzheimers Dement 2010; 6:230-238

13. Jack Jr CR, Bernstein MA, Borowski BJ, et al. Update on the magnetic resonance imaging core of the Alzheimer's disease neuroimaging initiative. Alzheimers Dement 2010; 6:212-220.

14. Reymer PW, Groenemeyer BE, van de Burg R, Kastelein JJ. Apolipoprotein E genotyping on agarose gels. Clinical chemistry 1995;41:1046-1047.

15. Hixson JE, Vernier DT. Restriction isotyping of human apolipoprotein E by gene amplification and cleavage with HhaI. Journal of lipid research 1990;31:545-548.

16. Hawkins JR, Khripin Y, Valdes AM, Weaver TA. Miniaturized sealed-tube allele-specific PCR. Human mutation 2002;19:543-553.

17. Myakishev MV, Khripin Y, Hu S, Hamer DH. High-throughput SNP genotyping by allele-specific PCR with universal energy-transfer-labeled primers. Genome research 2001;11:163-169.

18. Gibbons LE, Carle AC, Mackin RS, et al. A composite score for executive functioning, validated in Alzheimer's Disease Neuroimaging Initiative (ADNI) participants with baseline mild cognitive impairment. Brain Imaging Behav 2012; 6:517-527.

19. Crane PK, Carle A, Gibbons LE, et al. Development and assessment of a composite score for memory in the Alzheimer's Disease Neuroimaging Initiative (ADNI). Brain Imaging Behav 2012; 6:502-516.

20. Choi SE, Mukherjee S, Gibbons LE, et al. Development and validation of language and visuospatial composite scores in ADNI. Alzheimers Dement (N Y) 2020; 6: e12072.

21. Diabetes Screening Among Immigrants: A population-based urban cohort study Creatore MI, Booth GL, Manuel DG, Moineddin R, Glazier RH. Diabetes Care 2012; 35: 754-761.

22. Borroni B, Grassi M, Costanzi C, Archetti S, Caimi L, Padovani A. APOE genotype and cholesterol levels in lewy body dementia and Alzheimer disease: investigating genotype-phenotype effect on disease risk. Am J Geriatr Psychiatry 2006; 14:1022-1031.

23. Wang JC, Kwon JM, Shah P, Morris JC, Goate A. Effect of APOE genotype and promoter polymorphism on risk of Alzheimer's disease. Neurology 2000; 55:1644-1649.

24. Moreno-Grau S, Rodríguez-Gómez O, Sanabria Á, et al. Exploring APOE genotype effects on Alzheimer's disease risk and amyloid $\beta$ burden in individuals with subjective cognitive decline: The FundacioACE Healthy Brain Initiative (FACEHBI) study baseline results. Alzheimers Dement 2018; 14:634 643

25 McFall GP, Bäckman L, Dixon RA. Nuances in Alzheimer's Genetic Risk Reveal Differential Predictions of Non-demented Memory Aging Trajectories: Selective Patterns by APOE Genotype and Sex. Curr Alzheimer Res 2019; 16:302-315.

26. Albert M, Anja Soldan, Gottesman R, McKhann G, et al. Cognitive change preceding clinical symptom onset of mild cognitive impairment and 
relationship to ApoE genotype. Curr Alzheimer Res 2014; 11:773-784.

27. Negash S, Petersen LE, Geda YE, et al. Effects of ApoE genotype and mild cognitive impairment on implicit learning. Neurobiol Aging 2007; 28:885-893.

28. Knopman DS, Mosley TH, Catellier DJ, Coker LH. Fourteen-year longitudinal study of vascular risk factors, APOE genotype, and cognition: the ARIC MRI Study. Alzheimers Dement 2009; 5:207-214.

29. Seo EH, Kim SH, Park SH, Kang S, Choo IH. Independent and Interactive Influences of the APOE Genotype and Beta-Amyloid Burden on Cognitive Function in Mild Cognitive Impairment. J Korean Med Sci 2016; 31:286-295.

30. Christensen H, Batterham PJ, Mackinnon AJ, et al. The association of APOE genotype and cognitive decline in interaction with risk factors in a 65-69 year old community sample. BMC Geriatr 2008; 8:14.

31. Li B, Shi J, Gutman BA, et al. Influence of APOE Genotype on Hippocampal Atrophy over Time - An N=1925 Surface-Based ADNI Study. PLoS One 2016; 11:e0152901.

32. Roheger M, Kessler J, Kalbe E. Structured Cognitive Training Yields Best Results in Healthy Older Adults, and Their ApoE4 State and Baseline Cognitive Level Predict Training Benefits. Cogn Behav Neurol 2019;32:76-86. 